\title{
Papillary Thyroid Carcinoma with Malignant Pleural Effusion: Dilemma of Etiology
}

\author{
${ }^{1}$ Arvind Krishnamurthy, ${ }^{2}$ Siddappa KT \\ ${ }^{1}$ Associate Professor, Department of Surgical Oncology, Division of Head and Neck Oncology, Department of Surgical Oncology \\ Cancer Institute (WIA), Adyar, Chennai, Tamil Nadu, India \\ ${ }^{2}$ Postgraduate Student, Department of Surgical Oncology, Division of Head and Neck Oncology, Department of Surgical Oncology \\ Cancer Institute (WIA), Adyar, Chennai, Tamil Nadu, India
}

Correspondence: Arvind Krishnamurthy, Associate Professor in Surgical Oncology at Cancer Institute, 36, Sadar Patel Road Adyar, Chennai-20, Tamil Nadu, India, Phone: 9840448174, e-mail: drarvindkrishnamurthy@yahoo.co.in

\begin{abstract}
Papillary carcinomas of thyroid generally have an indolent course with metastatic disease in less than $5 \%$ of patients. Common sites of distant metastasis include lung and bone. A presentation with malignant pleural effusion is exceedingly rare with only a handful of cases reported in literature. We present a case and discuss the treatment of a patient presenting of papillary thyroid cancer with a relapse of the disease in the pleural cavity. There are no standard treatment recommendations in this setting in view of paucity of published data from literature.
\end{abstract}

Keywords: Thyroid cancer, papillary cancer, pleural effusion, metastasis.

\section{INTRODUCTION}

Papillary thyroid carcinoma usually metastasizes to the neck nodes, lung and bone but relapsing as a pleural effusion is rare. It is found in only $0.6 \%$ of patients as reported in a large case series over a period of 47 years. ${ }^{2}$ Only approximately 4 to $15 \%$ of patients presented initially with distant metastasis in well-differentiated thyroid cancer. ${ }^{3,8,9}$ We present a case report of one of our patients presenting as a malignant pleural effusion from papillary carcinoma thyroid. Metastatic pleural effusion as the initial manifestation in papillary thyroid cancer is reported very rarely. ${ }^{4}$

\section{CASE REPORT}

A 52-year-old gentleman, a chronic smoker with hypertension and ischemic heart disease on medication, presented to us in January 2006 with a thyroid swelling and right sided cervical lymphadenopathy. He was diagnosed to have papillary carcinoma thyroid. He underwent a total thyroidectomy, bilateral paratracheal lymph node dissection and right side modified radical neck dissection then. The final histopathology was reported as follicular variant of papillary carcinoma thyroid with extra thyroidal extension to the surrounding soft tissue, a total of 17/40 nodes was positive for metastasis. His postoperative Iodine 131 uptake was $0.1 \%$ and whole body scan was negative. He was kept under regular follow-up on eltroxin suppression. He presented to us in May 2009 with right side chest pain and dyspnea. His chest skiagram showed a massive right sided pleural effusion with a normal lung parenchyma (Fig. 1). Computed tomography (CT) of the chest confirmed the skiagram findings. A therapeutic thoracocentesis was done and pleural fluid was sent for biochemical analysis and cytology. It revealed an exudative effusion with the cytology

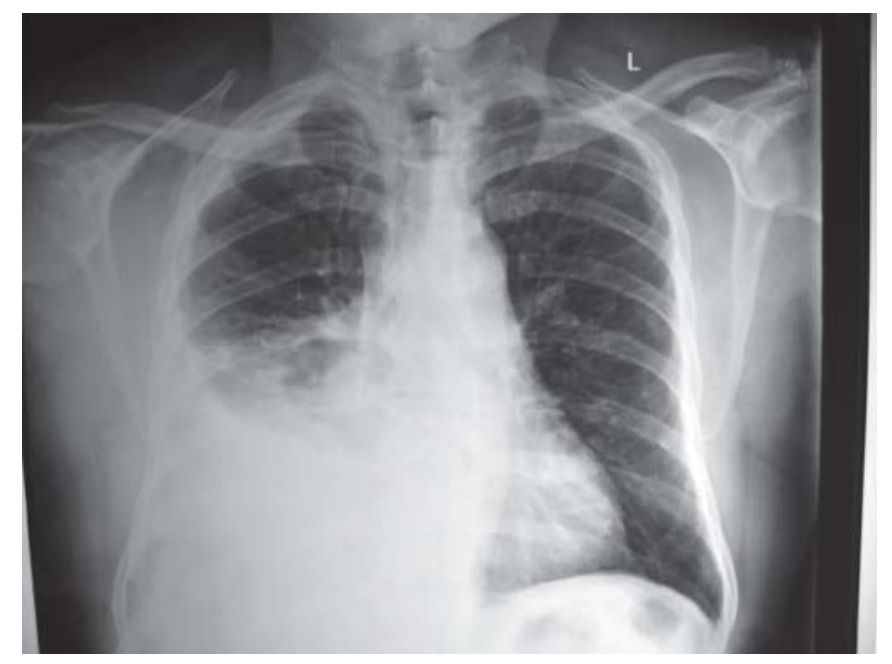

Fig. 1: Pleural effusion prior to plerodesis 


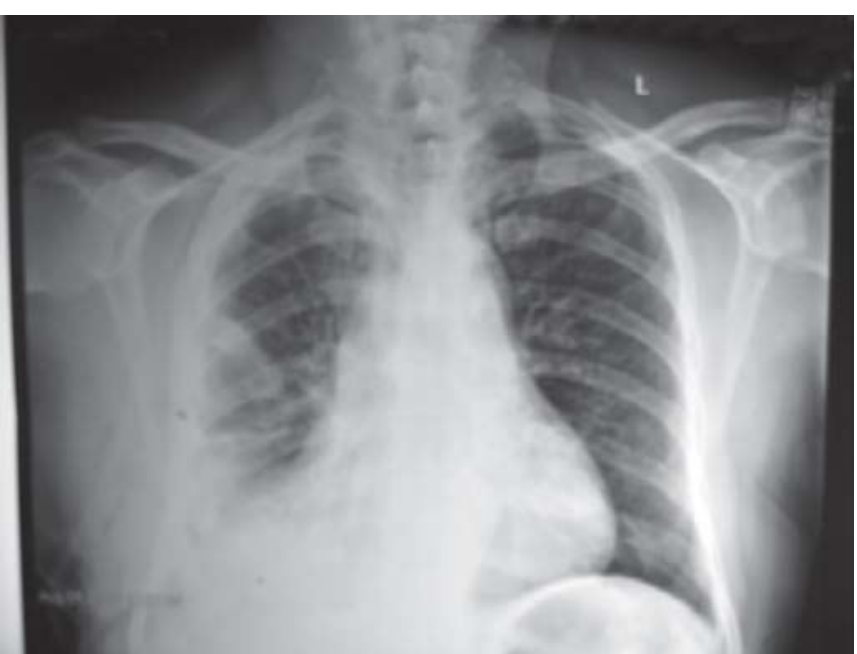

Fig. 2: Postpleurodesis

consistent with metastatic papillary thyroid carcinoma. His serum thyroglobulin was $54.3 \mathrm{ng} / \mathrm{ml}$, an Iodine 131 uptake scan was $0.3 \%$ and whole body scan was negative. In view of nonfunctioning metastasis, radio-iodine therapy was not considered. A tetracycline pleurodesis was done (Fig. 2). The patient was not inclined to consider any other treatment options (chemotherapy/targeted therapy), there being no standard recommendations and is following up with us on eltroxin suppression.

\section{DISCUSSION}

Malignant pleural effusions are caused most commonly by carcinomas of the breast, lung, gastrointestinal tract, ovary and lymphoid malignancies. In male patients about half of malignant effusions are caused by lung cancer, $20 \%$ by lymphomas or leukemia, $7 \%$ from gastrointestinal primaries, $6 \%$ from genitourinary primaries, and $11 \%$ from tumors of unknown primary site. In female patients, about $40 \%$ of malignant effusions are caused by breast cancer, $20 \%$ from tumors arising in the female genital tract, $15 \%$ from lung primaries, $8 \%$ from lymphomas or leukemia, $4 \%$ from gastrointestinal tract primaries, $3 \%$ from melanoma, and $9 \%$ from tumors of unknown primary site. ${ }^{1}$

Papillary carcinomas of thyroid generally have an indolent course with metastatic disease in less than $5 \%$ of patients. Common sites of distant metastasis include lung $(67 \%)$ and bone (25\%). A presentation with malignant pleural effusion is exceedingly rare. Immunohistochemistry with TTF-1 and thyroglobulin on cytology helps to

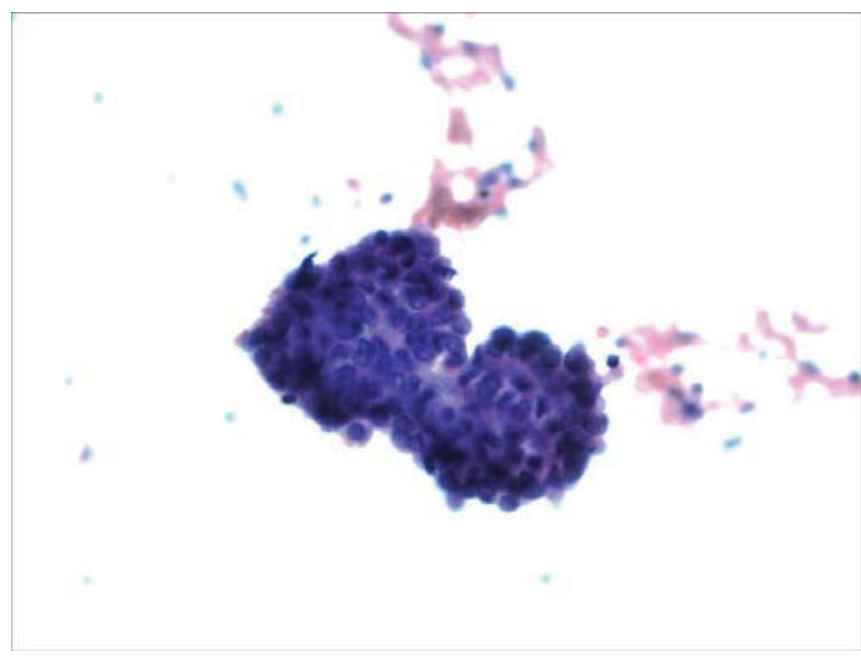

Fig. 3: Pleural fluid in papillary carcinoma thyroid

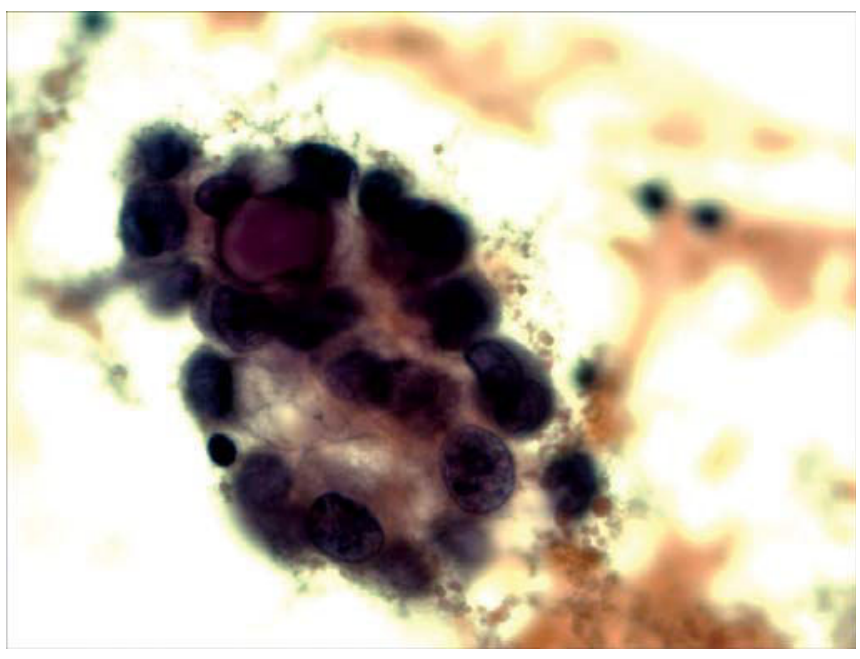

Fig. 4: Psammoma body in plerual fluid cytology

differentiate metastatic adenocarcinoma lung from thyroid where in serum thyroglobulin is normal. ${ }^{7}$ Typically, the presence of a malignant pleural effusion portends a dismal prognosis, with a reported median survival of 11 months. ${ }^{2,4}$ Metastatic pleural effusion commonly seen in papillary carcinoma thyroid except one case report of hurtle cell carcinoma (Figs 3 and 4). 5,6

\section{CONCLUSION}

Malignant pleural effusion from a papillary carcinoma is an exceedingly rare differential diagnosis even in the background of thyroid malignancy. In view of paucity of data there are no standard treatment recommendations. 


\section{REFERENCES}

1. Johnston WW. The malignant pleural effusion: A review of cytopathologic diagnoses of 584 specimens from 472 consecutive patients. Cancer 1985;56(4):905-09.

2. Vassilopoulou-Sellin R, Sneige N. Pleural effusion in patients with differentiated papillary thyroid cancer. South Med J 1994;87(11):1111-16.

3. Shaha AR, Shah JP, Loree TR. Differentiated thyroid cancer presenting initially with distant metastasis. Am J Surg 1997; 174:474-76.

4. Jung KH, et al. Papillary thyroid cancer with malignant pleural effusion. Tuberculosis and Respiratory Diseases Apr. 2008;64(4).

5. Andrew NV, Leslie R Sheeler, Charles V Biscotti, James K Stoller. Pleural effusion resulting from metastatic papillary carcinoma of the thyroid. Chest 1992;101:1448-50.
6. Kuo-Feng Hsu, Chung-Bao Hsieh, Quan-Yang Duh, Chih-Feng Chien, Heng-Sheng Li, Ming-Lang Shih. Hürthle cell carcinoma of the thyroid with contralateral malignant pleural effusion. Onkologie 2009;32:47-49.

7. Mundathaje, et al. Albert Einstein Medical Center, Philadelphia. Papillary carcinoma of thyroid recurring as pleural effusion after 26 years after curative therapy. Chest 2006; 130(4):331S.

8. Clark JR, Lai P, Hall F, Borglund A, Eski S, Freeman JL. Variables predicting distant metastases in thyroid cancer. Laryngoscope 2005; 15:661-67.

9. Casara D, Rubello D, Saladini G, et al. Different features of pulmonary metastases in differentiated thyroid cancer: Natural history and multivariate statistical analysis of prognostic variables. J Nucl Med 1993;34:1626-31. 